\title{
Distributed Secondary Frequency and Voltage Control of Parallel-Connected VSCs in Microgrids: A Predictive VSG-Based Solution
}

\author{
Oluleke Babayomi, Zhen Li, and Zhenbin Zhang
}

\begin{abstract}
The need for converter-based synthetic inertia has become more important due to the increasing level of renewable generation penetration in power systems. In this paper, the distributed secondary regulation of frequency and voltage is implemented for model predictive-controlled (MPC) voltage source converters (VSCs) in an AC microgrid (MG). A virtual synchronous generator (VSG) provides inertia-emulation to reduce the rate of change of frequency (ROCOF) that arises from sudden load changes. First, a small-signal stability analysis for parallel-connected VSG-based inverters in a MG is analyzed. Next, the secondary control of voltage and frequency in a distributed AC MG (with parallel-connected inertia-emulating VSCs) is realized. In addition, for the applied load changes in this study it is shown that the proposed control scheme effectively reduces the load change-induced ROCOF by up to $89 \%$ and also has very fast and accurate dynamic response that supports robust and rapid recovery from perturbations to MG stability.
\end{abstract}

Index Terms-AC microgrid, inertia emulation, model predictive control, power converter, rate of change of freguecy (ROCOF), smallsignal stability, virtual synchronous generator.

\section{INTRODUCTION}

$\mathrm{T}$ HE inertia of an electrical power system is a measure of how much resistance it poses to external disturbances that want to influence its steady state. Therefore, the ideal power system is one that has infinite inertia and whose state cannot be altered by transient disturbances [1]. In practical situations, the closest we have to the ideal is the power grid (stiff grid). Whenever there is a rise or drop in power demand by connected consumers, the power grid can adjust to accommodate

Manuscript received July 15, 2020; revised September 10, 2020; accepted September 15, 2020. Date of publication December 30, 2020; date of current version December 9, 2020. This work was financially supported in part by the Shandong Provincial Key Research and Development Program (Major Scientific and Technological Innovation Project under Grant 2019JZZY020805, in part by the National Distinguished Expert (Youth Talent) Program of China under Grant 31390089963058, in part by the General Program of National Natural Science Foundation of China under Grant 51977124, in part by the Shandong Natural Science Foundation under Grant ZR2019QEE001, and in part by the Natural Science Foundation of Jiangsu Province under Grant BK20190204. (Corresponding author: Zhenbin Zhang.)

All authors are with the School of Electrical Engineering, Shandong University, Jinan 250061, China (email: oluleke.babayomi@mail.sdu.edu.cn; zhenli0901@sdu.edu.cn; zbz@sdu.edu.cn).

Digital Object Identifier 10.24295/CPSSTPEA.2020.00028 such changes without any significant effect on the steady state conditions. The inertia of a power system, supported by rotating synchronous generators and turbines, stabilizes the system operation by sensing the frequency variation and initiating a corresponding increase or decrease in the angular speed of these rotating machines.

On the other hand, in modern power systems that are underpinned by large-scale renewable generation, the mechanism for power system stability requires innovative solutions. This is because rotating physical machines are being replaced by renewable static generators interfaced with power electronic converters; the latter introduce a situation of lower system inertia, accompanied with higher vulnerabilities to sudden load changes. In more recent times, power electronics converters are playing a helpful role in ameliorating this challenge. Inertiabased power electronics can be categorized into two: inertiabased power converters [1], [2] (for wind turbines and virtual synchronous generators/motors) and inertia-supporting energy storage systems [3]-[5] (batteries, DC-link capacitors and super-capacitors).

In inertia-based power converters, virtual inertia - in a virtual synchronous generator (VSG) - is amenable to synthesis in order to improve the mimicry capabilities of the VSG to mitigate frequency changes that arise from power transients. This design process can be achieved using self-tuning schemes [6]. A finite control set model predictive controlled (FCS-MPC) fault ride-through scheme for an inverter was proposed in [7]. The control is VSG-based and provides over-current protection to the distribution generation (DG) inverter during system fault condition; however, stability studies were not covered.

The ability of grid-forming inverters to provide system reference voltage and provide inertial support to the microgrid (MG) was the subject of [8]. The study compared the performance of centralized and decentralized MPC schemes in achieving the goals of reducing frequency nadir and rate of change of frequency (ROCOF). The developed scheme was validated on an IEEE 39-bus system. An investigation into the frequency stability of low inertia MGs was reported in [9]. Using ramp-rate constraints, MPC was used to optimize frequency control to achieve peak output power requirements and reduce the system's ROCOF. These studies also excluded small-signal analysis.

The centralized model predictive-controlled (MPC) scheme 
[10] operates in grid-forming and grid-following mode. Its weaknesses are the difficulty of scale and single-point-offailure risk. The decentralized topology [11]-[13] also has limits to scalability and so can work for relatively smallscale MGs. The distributed control topologies surveyed [9], [14], [15], offer more promising reliability and flexibility than centralized and decentralized control schemes. Thus, the distributed architecture has been selected for further study in this work.

Since droop-based primary control in MGs has challenges with voltage and frequency deviations whenever there are load changes, effective secondary regulation becomes necessary. A secondary control system was proposed in [16] to restore the frequency considering the communication delays. This research considered two types of controllers i.e., controller based on Smith Predictor and MPC. MPC was proposed to implement secondary regulation to mitigate stability issues arising from communication delays. MPC-based secondary control demonstrates more robust performance and faster dynamic performance than proportional-integral (PI) and Smith predictor-based secondary control. Hence, this control family was recommended for a system where communication delay is unknown with large variations [16]. Nonetheless, none of the above-mentioned studies incorporated inertia emulation into the VSC-system used for primary control. There is also a lack of small-signal analysis for parallel-connected VSG-based inverters in the literature.

Therefore, the objective of this paper is the inertia-enhanced secondary frequency and voltage regulation of VSCs in an $\mathrm{AC}$ microgrid. The advantages of inertia emulation in VSG as well as the fast and robust control properties of MPC are being harnessed to enhance the response of secondary control. The specific contributions of this paper are namely:

1) The small-signal analytic studies on the stability of parallel-connected inertia-emulated VSCs in an islanded AC MG are presented. Although the small-signal model for a VSG was reported in [17], only the standalone case for a single VSG was studied. This paper extends the results presented in [17], [18] to the case of parallelconnected inertia-emulating VSCs in an AC MG.

2) The secondary control of voltage and frequency in a distributed AC MG (with parallel-connected inertia emulated VSCs) is realized and discussed.

3) The proposed control scheme effectively reduces the high ROCOF associated with the primary control of the VSCs.

The rest of the paper is organized as follows: Section II reviews the equivalence of the MG droop control and VSG inertia emulation. In Section III, the small-signal stability analysis of inverters controlled by VSG-emulated droop is evaluated. Distributed MG converter control based on MPC is introduced in Section IV. Simulation results are presented and discussed in Section V, and the conclusion is drawn in Section VI.

\section{Equivalence of Microgrid Droop AND Virtual INERTIA EMULATION}

In this section, the relationship between microgrid droop and virtual inertia emulation will be briefly stated; a basis for further discussions in the rest of the paper.

\section{A. The Swing Equation}

The inertia emulation of a synchronous machine via the VSG is implemented through the swing equation, viz.,

$$
J \frac{\mathrm{d} \omega}{\mathrm{d} t}=T_{\mathrm{m}}-T_{\mathrm{e}}-k_{d}\left(\omega-\omega^{*}\right)
$$

where $\omega$ is the machine angular frequency, $k_{d}$ is the damping coefficient of the damper windings, $\omega^{*}$ is the synchronous frequency and $J$ is the rotor's moment of inertia.

From (1), the swing equation can be expressed in terms of the inertia constant $(\mathrm{H})$ and power (in frequency domain) as

$$
2 \mathrm{H} \omega s=P_{\mathrm{m}}-P-k_{d}\left(\omega-\omega^{*}\right),
$$

where the $P_{\mathrm{m}}$ and $P$ are mechanical and electrical power of the machine respectively. Also, the inertia constant is related to moment of inertia through, $J=2 \mathrm{H} S_{\text {rated }} / \omega^{2}$. The machine governor is modeled with

$$
P_{\mathrm{m}}=P_{\mathrm{n}}-k_{p}\left(\omega-\omega^{*}\right)
$$

where $k_{p}$ is the $\omega-P$ droop coefficient.

\section{B. Microgrid Droop Equations}

The droop relationship, expressed via the following equations, is the conventional method for controlling frequency and voltage through the active and reactive powers of inverters, viz.:

$$
\begin{aligned}
\omega & =\omega^{*}-k_{p}\left(\tilde{P}-P^{*}\right), \\
V & =V^{*}-k_{v}\left(\tilde{Q}-Q^{*}\right),
\end{aligned}
$$

where $k_{p}$ and $k_{v}$ are MG droop coefficients for frequencyactive-power and voltage-reactive-power respectively. The low-pass filters (LPF) used prior to power measurement account for the definitions of $\tilde{P}$ and $\tilde{Q}$ as

$$
\begin{aligned}
& \tilde{P}=\frac{\omega_{\mathrm{f}}}{s+\omega_{\mathrm{f}}} P, \\
& \widetilde{Q}=\frac{\omega_{\mathrm{f}}}{s+\omega_{\mathrm{f}}} Q,
\end{aligned}
$$

where $\omega_{\mathrm{f}}$ is the LPF cut-off frequency. Substituting (6) and (7) into (4),

$$
\omega=\omega^{*}-k_{p}\left[\frac{\omega_{\mathrm{f}}}{s+\omega_{\mathrm{f}}} P-P^{*}\right]
$$

By making $P$ subject of (8) and equating derivatives of constants to zero, 


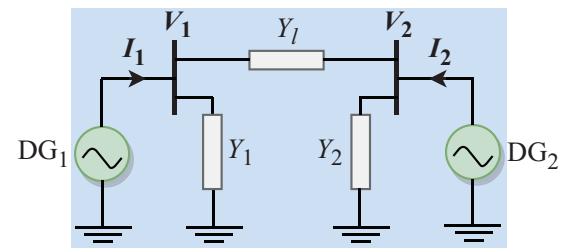

Fig. 1. A microgrid system with two DGs interconnected by a power line.

$$
\frac{\omega \cdot s}{\omega_{\mathrm{f}} k_{p}}=P^{*}-P+\frac{1}{k_{p}}\left(\omega^{*}-\omega\right) .
$$

A comparison of (2) and (9), results in the parameters for VSG emulation as

$$
H=\frac{1}{2 \omega_{\mathrm{f}} k_{p}}, k_{d}=\frac{1}{k_{p}}
$$

\section{Small-Signal and Frequency Stability Analyses}

In this section, the analytic formulations for VSG-emulated inverter and microgrid system small-signal stability analyses will be derived. The method introduced in [18] is extended to the case of paralled-connected VSG-based inverters.

\section{A. VSG-Based Inverter Small-Signal Analysis}

The method in [18] is extended to VSG-inverters in the following discussion. After appropriate substitutions in (4) and (5), and linearizing for small perturbations about the nominal values, the following result ensues for the VSG small-signal model for frequency-active power and voltagereactive power:

$$
\left[\begin{array}{c}
\Delta \dot{\omega} \\
\Delta \dot{v}_{d} \\
\Delta \dot{v}_{q}
\end{array}\right]=\boldsymbol{E}\left[\begin{array}{l}
\Delta \omega \\
\Delta v_{d} \\
\Delta v_{q}
\end{array}\right]+\boldsymbol{F}\left[\begin{array}{c}
\Delta P \\
\Delta Q
\end{array}\right]
$$

where $v_{d}, v_{q}$ are $d$ - and $q$-axes voltage components, respectively; $\boldsymbol{E}, \boldsymbol{F}$ are defined in Appendix B.

\section{B. Microgrid System Small-Signal Stability}

The generic microgrid system of interconnected VSCs has dynamics described in (12); $\boldsymbol{A}$ is the system's state matrix, and $\boldsymbol{X}$ is the matrix of state variables.

$$
\dot{\boldsymbol{X}}=\boldsymbol{A} \boldsymbol{X}
$$

The case of two parallel-connected VSCs that have voltagecontrolled VSG-emulation capabilities to improve system inertia is shown in Fig. 1. Details on the derivation of the smallsignal model of this MG system are provided in Appendix C.

The oscillations of the output variables are conditioned by the state matrix $\boldsymbol{A}$. Thus, the non-zero eigen values would be
TABLE I

Parameters for Small Signal Analysis

\begin{tabular}{lcc}
\hline \hline Parameters & Symbols & Values \\
\hline Nominal frequency & $\omega_{\mathrm{n}}$ & $377 \mathrm{rad} / \mathrm{s}$ \\
LPF cut-off frequency & $\omega_{\mathrm{f}}$ & $37.7 \mathrm{rad} / \mathrm{s}$ \\
Impedances & $Z_{1}, Z_{1}, Z_{2}$ & $Z_{1}=(0.2+j 3) \Omega$ \\
& & $Z_{1}=(13+j 6) \Omega$, \\
& & $Z_{2}=(25+j 13) \Omega$ \\
Sources & $S_{1}, S_{2}$ & $S_{1}=(806+j 384) \mathrm{VA}$ \\
& & $S_{2}=(750+j 375) \mathrm{VA}$ \\
Voltage sources & $V_{1}, V_{2}$ & $V_{1}=127 \angle 0^{\circ} \mathrm{V}$, \\
& & $V_{2}=130 \angle 2.1^{\circ} \mathrm{V}$ \\
Droop coefficients & $k_{p}, k_{v}$ & $k_{p}=k_{v}=5 e^{-3}$ \\
\hline \hline
\end{tabular}

analyzed for their sensitivity to parameters variations based on parameters provided in Table I. Fig. 2(a) and (b) compares the effect of varying the damping constant $k_{\mathrm{d}}$ at both low and high line inductances. It is seen that there are many eigen values on the right-hand real axis when inductance is low (Fig. 2(b)). On the other hand, when the inductance is high, all the eigen values lie on the left-hand side, indicating stability. Thus, higher power line inductance improves stability. Virtual impedance can be used to improve the line reactance (hence, inductance) [17]. Fig. 2(c) and (d) shows that $k_{\mathrm{d}}$ moves the eigen values closer to the real axis, reducing oscillation frequency. Fig. 2(e) and (f) illustrates the eigenvalues as line inductance changes for low inertia (Fig. 2(e)) and high inertia (Fig. 2(f)). It is seen that low inertia results in many of the eigenvalues being very far from the real axis (up to $180 \mathrm{rad} / \mathrm{s}$ ), imparting high frequency oscillations to the system response. However, when inertia is high, the majority of oscillation frequencies are less than $2 \mathrm{rad} / \mathrm{s}$, with many of them close to the origin or at the origin. Thus, the results of this stability studies corroborate the findings of [17], [18].

In order to examine the transient behavior of the multimachine system (Fig. 1), we can draw insights from Fig. 3. First, when the DG equivalent reactances are equally matched $\left(X_{1}=X_{2}\right)$, the transient characteristics comprise mainly of decaying exponentials, void of oscillations and is overdamped (slower decay of transients). Next, as the DG reactance mismatches increase from 0.5 times up to the value of $X_{1}$, the eigen values have an increasing sinusoidal characteristic. In practical terms, this means that when we desire proportional reactive power sharing in mismatched VSG-based VSCs, the practice of using virtual impedances [19] to match DG impedances could come at the expense of slower transient decay.

\section{Distributed MG Converter Control}

In this section, the concepts underlying MPC will first be discussed. Subsequently, the control scheme for distributed secondary regulation of AC MG frequency and voltage will be formulated.

\section{A. Model Predictive Inverter Control}

MPC facilitates the multivariable control of power electronic 


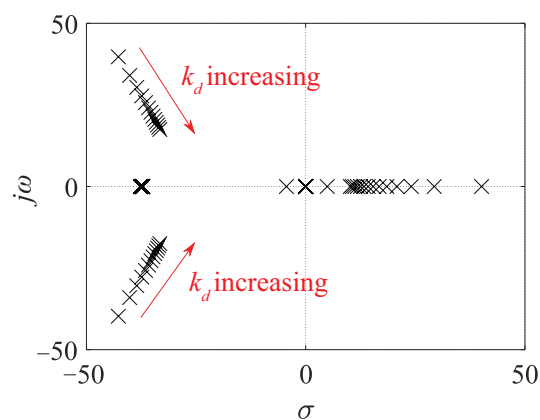

(a) $L_{1}=0.1 \mathrm{mH}$

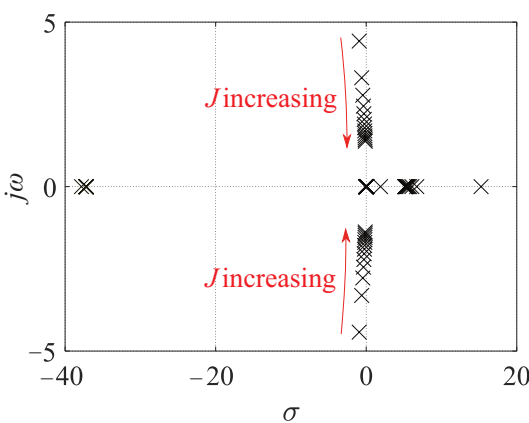

(d) $L_{1}=1 \mathrm{mH}$

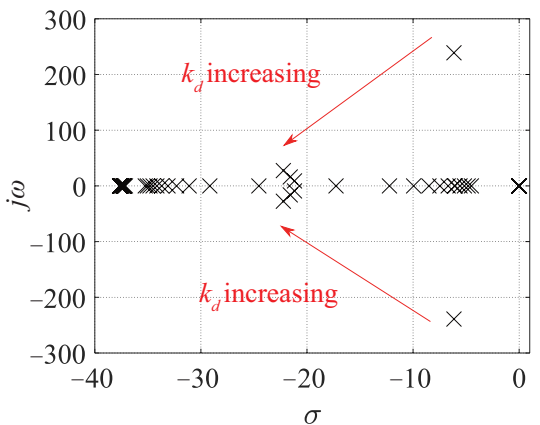

(b) $L_{1}=1 \mathrm{mH}$

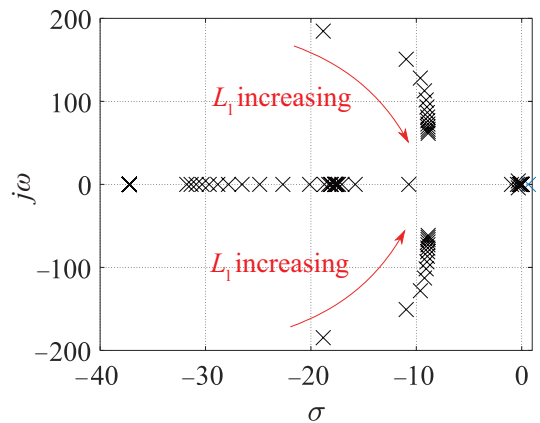

(e) $J=1.5 e^{-4} J_{0}$

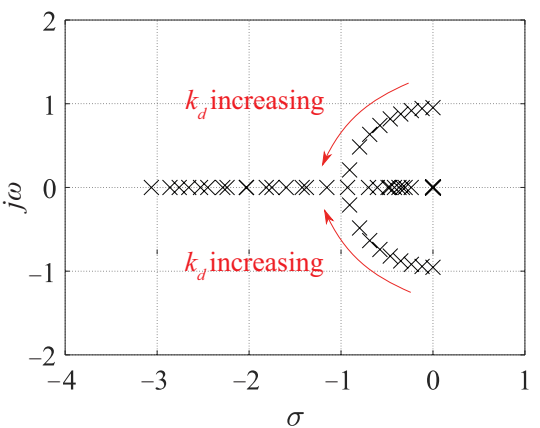

(c) $J=1.5 e^{5} J_{0}$

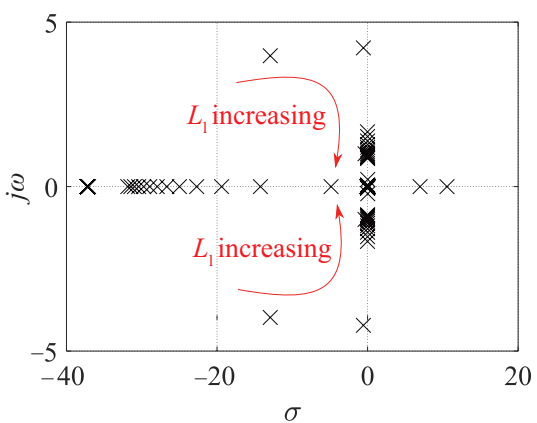

(f) $J=1.5 e^{5} J_{0}$

Fig. 2. Root locus plots. (a) $k_{d}$ of VSG varies from $1.5 e^{-6} k_{d 0}$ to $1.5 e^{3} k_{d 0}$, at $L_{1}=0.1 \mathrm{mH}$. (b) $k_{d}$ of VSG varies from $1 e^{-6} k_{d 0}$ to $1 e^{3} k_{d 0}$, at $L_{1}=1 \mathrm{mH}$. (c) $k_{d}$ of VSG varies from $1.5 e^{-6} k_{d 0}$ to $1.5 e^{3} k_{d 0}$, at $1.5 e^{5} J_{0}$. (d) $J_{0}$ of VSG varies from $1.5 e^{-4} J_{0}$ to $1.5 e^{5} J_{0}$, at $L_{1}=1 \mathrm{mH}$. (e) $L_{1}$ of line varies from $0.1 \mathrm{mH}$ to $10 \mathrm{mH}$, at $1.5 e^{-4} J_{0}$. (f) $L_{1}$ of line varies from $0.1 \mathrm{mH}$ to $10 \mathrm{mH}$, at $1.5 e^{5} J_{0}$.

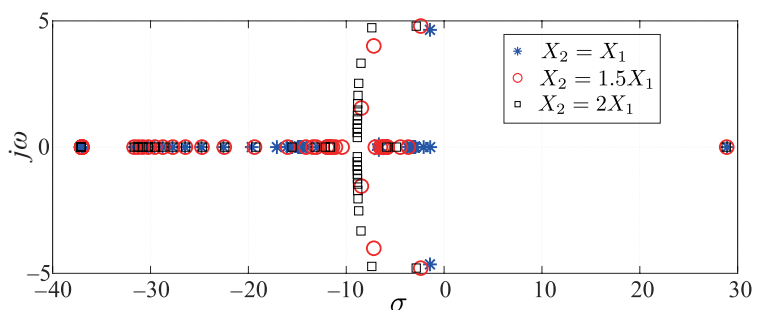

Fig. 3. Loci of the eigen values for the transient characteristics of multimachine VSG-based VSC microgrids. The inductance $L_{1}$ of the line varies from $0.1 \mathrm{mH}$ to $10 \mathrm{mH}$ at $1.5 e^{5} J_{0}$.

switching devices while accommodating numerous constraints that are peculiar to different situations. Clarke's transformation is applied to obtain the $\alpha \beta$ transformation of the current and voltage vectors according to the equation

$$
\bar{x}_{\alpha}+j \bar{x}_{\beta}=\boldsymbol{K}\left[\begin{array}{lll}
x_{\mathrm{a}} & x_{\mathrm{b}} & x_{\mathrm{c}}
\end{array}\right]^{\prime},
$$

where $K=\frac{1}{3}\left[1 e^{j \frac{2}{3} \pi} e^{j \frac{4}{3} \pi}\right]$. By Kirchoff's current and voltage laws, the LC filter is modeled in state-space as

$$
\frac{\mathrm{d}}{\mathrm{d} t}\left[\begin{array}{c}
\bar{i}_{\mathrm{f}} \\
\bar{v}_{\mathrm{f}}
\end{array}\right]=\boldsymbol{B}\left[\begin{array}{c}
\bar{i}_{\mathrm{f}} \\
\bar{v}_{\mathrm{f}}
\end{array}\right]+\boldsymbol{C}\left[\begin{array}{c}
\bar{v}_{\mathrm{i}} \\
\bar{i}_{\mathrm{o}}
\end{array}\right],
$$

where $B=\left[\begin{array}{cc}-\frac{R_{\mathrm{f}}}{L_{\mathrm{f}}} & -\frac{1}{L_{\mathrm{f}}} \\ \frac{1}{C_{\mathrm{f}}} & 0\end{array}\right] ; \mathrm{C}=\left[\begin{array}{cc}\frac{1}{L_{\mathrm{f}}} & 0 \\ 0 & -\frac{1}{C_{\mathrm{f}}}\end{array}\right]$.
The variables $R_{\mathrm{f}}, L_{\mathrm{f}}$ and $C_{\mathrm{f}}$ represent the filter resistance, inductance and capacitance respectively. Also, $\bar{i}_{\mathrm{f}}$ and $\bar{v}_{\mathrm{f}}$ are filter current and voltage respectively.

The control objectives are to ensure that the appropriate input voltage is applied to the VSC so that the output voltage accurately follows the desired reference. These are achieved by the following cost function

$$
G=\sum_{k}^{k+N-1}\left[\left\|-^{*}(k)-\overline{v_{\mathrm{f}}}(k)\right\|^{2}+\psi_{\lim }(k)+\chi S W^{2}(k)\right],
$$

where the first term is the voltage prediction error, the second term accounts for the physical current limits on the device and the last term penalizes the switching effort with weighting factor $\chi$. The latter two terms are expressed as

$$
\begin{aligned}
& \psi_{\text {lim }}(k)= \begin{cases}0 & \text { if }\left|i_{\mathrm{f}}(k)\right| \leqslant i_{\text {max }}, \\
\infty & \text { if }\left|i_{\mathrm{f}}(k)\right| \leqslant i_{\text {max }},\end{cases} \\
& S W(k)=\sum|u(k)-u(k-1)| .
\end{aligned}
$$

\section{B. MPC Frequency Analysis}

The frequency stability analysis of MPC is usually carried out using a describing function [20], [21]. This process involves introducing perturbation signals at the input of the inner voltage loop and recording the corresponding output voltages. These signal comprise a series of equal amplitude inputs at varying frequencies (from $50 \mathrm{~Hz}$ to $5 \mathrm{kHz}$ ). Comparing this result with 


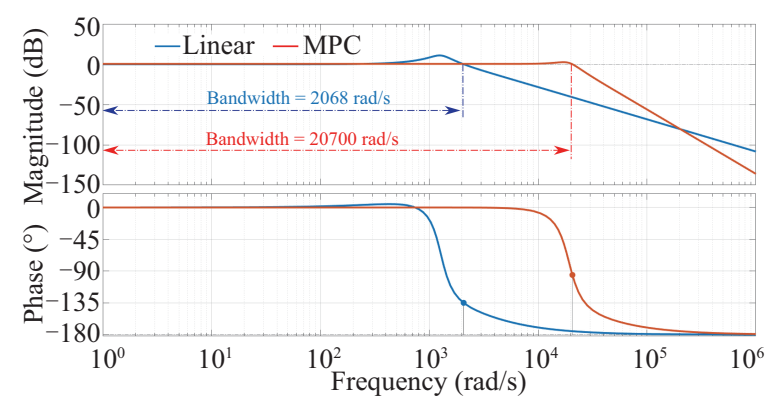

Fig. 4. Bode plots for MPC and linear voltage control of VSC.

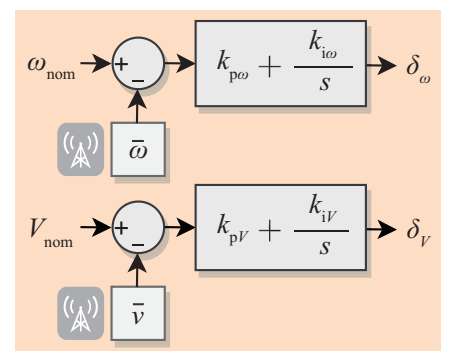

Fig. 5. Distributed secondary control for frequency and voltage.

the output of a linear voltage controller (proportional-resonant controller) [22], the Bode plot in Fig. 4 ensues. The frequency characteristic of MPC indicates that it provides a higher voltage-loop bandwidth than linear control-20700 rad/s vs. $2068 \mathrm{rad} / \mathrm{s}$ respectively.

\section{Distributed Secondary Control}

Secondary control is deployed to regulate and compensate for the drift that occurs whenever there is a change in active or reactive power level. The drift arises from droop control following the droop slope for the inverter in consideration. Fig. 5 illustrates the proposed PI-controller based secondary control. The secondary level introduces the following references for voltage and frequency

$$
\begin{aligned}
& \omega_{\text {ref }}=\omega_{\text {nom }}-k_{p} P+\left(K_{\mathrm{p} \omega}+\frac{K_{\mathrm{i} \omega}}{s}\right)\left(\omega_{\text {nom }}-\omega\right), \\
& V_{\text {ref }}=V_{\text {nom }}-k_{v} Q+\left(K_{\mathrm{p} V}+\frac{K_{\mathrm{i} V}}{s}\right)\left(V_{\text {nom }}-V\right),
\end{aligned}
$$

where $\omega_{\text {nom }}, V_{\text {nom }}$ are the nominal frequency and voltage respectively; $K_{\mathrm{p} \omega}, K_{\mathrm{p} V}, K_{\mathrm{i} \omega}$ and $K_{\mathrm{i} V}$ are the proportional and integral coefficients for frequency and voltage respectively.

Distributed secondary control is usually preferred for practical applications due to the ease of scalability and the possibilities of plug-and-play characteristics inherent in the architecture. Centralized controllers suffer from the limitation of total system collapse if the MG central controller has a downtime. Thus, the frequency and voltage signals that are shared across the communication channel for the control of each VSC out of a total $N$ are the averages in (20) and (21) respectively.

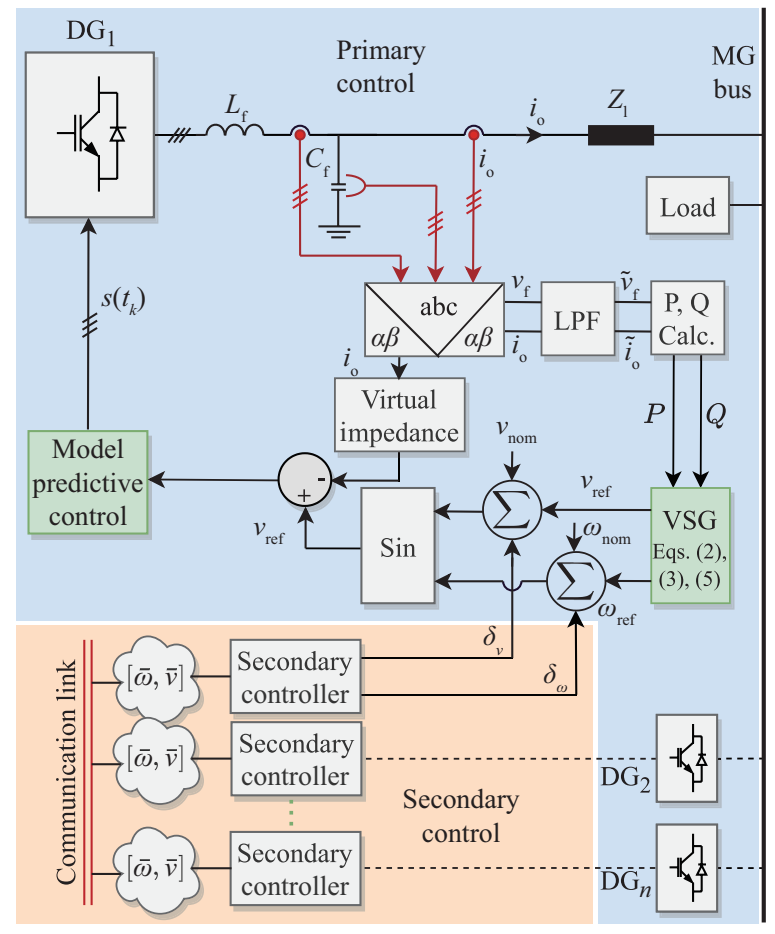

Fig. 6. Microgrid system for inertia-enhanced distributed MPC-based control [23].

$$
\begin{aligned}
& \bar{\omega}=\frac{\sum_{k=1}^{N} \omega_{D G_{k}}}{N}, \\
& \bar{V}=\frac{\sum_{k=1}^{N} V_{D G_{k}}}{N},
\end{aligned}
$$

Introducing MPC to the VSC has inherent characteristics of increasing the speed and bandwidth of control at the primary level. Therefore, there is a faster range of response possible to the secondary control. In conventional methods, linear lowbandwidth controllers are used at the primary and secondary levels. However, these slow the response of the system. Therefore, the VSC at each DG in this study is embedded with the capability for both high-speed response and improved inertia-emulation via the inertia-emulation principles earlier described. The test carried out in this section is based on the system architecture shown in Fig. 6. The primary control and secondary control sections are clearly seen. As highlighted before, a passive LC filter is applied to filter the output current harmonics, especially the high frequency and switching frequency harmonics.

\section{RESULTS AND Discussion}

As was earlier mentioned in Section I, this paper proposes the application of the emulated virtual inertia of the VSG to improve the frequency response of an MG system. At the same time, it applies MPC to the control of the VSC, to provide multi-variable robust and fast voltage control to an islanded MG. The concept is depicted in Fig. 6. At the primary 
TABLE II

Parameters Used for the Simulation of the Test Microgrid System

\begin{tabular}{lcc}
\hline \hline Parameters & Symbols & Values \\
\hline DC voltage & $V_{\mathrm{dc}}$ & $500 \mathrm{~V}$ \\
Nominal frequency & $f_{\mathrm{nom}}$ & $50 \mathrm{~Hz}$ \\
Nominal voltage & $V_{\mathrm{nom}}$ & $250 \mathrm{~V}$ \\
Filter & $R_{\mathrm{f}}, L_{\mathrm{c}}$ & $R_{\mathrm{f}}=0.1 \Omega$ \\
& $C_{\mathrm{f}}$ & $L_{\mathrm{f}}=2.4 \mathrm{mH}$ \\
& & $C_{\mathrm{f}}=15 \mu \mathrm{F}$ \\
Sampling time & $f_{\mathrm{s}}$ & $25 \mu \mathrm{s}$ \\
Droop coefficients & $k_{p}, k_{v}$ & $k_{p}=(2 \cdot 1 \mathrm{e}-3) \mu \mathrm{V} / \mathrm{W}$, \\
& & $k_{v}=(5 \cdot 2 \mathrm{e}-3) \mu \mathrm{rad} / \mathrm{sVar}$ \\
Line impedance & $R_{1}, L_{1}$ & $R_{1}=0.1 \Omega$, \\
& & $L_{1}=1.85 \mathrm{mH}$ \\
Virtual impedance & $R_{\mathrm{V}}, L_{\mathrm{v}}$ & $R_{\mathrm{v}}=0.2 \Omega$, \\
& & $L_{\mathrm{v}}=4 \mathrm{mH}$ \\
LPF cut-off frequency & $\omega_{\mathrm{f}}$ & $0.15 \mathrm{kHz}$ \\
Inertia & $J_{0}$ & $56.3 \mathrm{~kg} / \mathrm{m}^{2}$ \\
Secondary PI & $K_{\mathrm{p} \omega}, K_{\mathrm{i} \omega}$ & $K_{\mathrm{p} \omega}=150, K_{\mathrm{i} \omega}=3 e^{-2}$ \\
& $K_{\mathrm{p} v}, K_{\mathrm{i} v}$ & $K_{\mathrm{p} v}=40, K_{\mathrm{i} v}=10 e^{3}$ \\
\hline \hline
\end{tabular}

control level, virtual impedance is used to modify the output impedance of the VSC to a dominantly inductive one. This is beneficial to emerging power systems (comprising DGs which power MGs in close proximity) with less inductive power lines. The VSG-based droop, as governed by (2), (3), and (5) produce primary reference voltage $V_{\text {ref }}$ and frequency $\omega_{\text {ref }}$. These are corrected with compensatory signals $\delta_{v}$ and $\delta_{\omega}$ respectively, from the secondary distributed controller. The operational principles of the distributed secondary controller are expatiated in Section IV-C. The results of the simulation in MATLAB/Simulink, of the proposed scheme, are high-lighted in the discussion that follows. These outcomes are derived for MG and VSG-based VSC parameters provided in Table II.

\section{A. Equal Power Sharing}

Fig. 7 shows the system's response to a step-increase change in power at $0.4 \mathrm{~s}$ and step-decrease at $0.7 \mathrm{~s}$. This applies to the case of two inverters with equal parameters and connected to the common bus via equal line impedances. It can be seen in Fig. 7(a) and (b) that active and reactive power sharing respectively, in the MPC-based droop and VSG controllers are similar in performance for the equally rated inverters. Also, after the step change in load, both the active and reactive powers settled at steady state with a rapid rise time of $20 \mathrm{~ms}$. MPC VSC control is responsible for rapid response to load changes.

\section{B. Unequal Power Sharing}

Active power sharing is usually accurate for unmatched line impedances, but it becomes inaccurate for reactive power sharing. This can be corrected by the use of enhanced virtual impedance described in [19], [24]. Using this approach, we also made the inertia for inverter 1 and inverter 2 different, $J_{1}: J_{2}$ is $2: 1$; the ratings of the inverters were changed to alter their droop slopes, $S_{1}: S_{2}$ is $1: 3$. These resulted in the performance depicted in Fig. 8. The proportional sharing of both active and reactive powers was sustained during transient and steady states, although the reactive power had a delay in returning to steady state after the second step-change at $0.7 \mathrm{~s}$.

\section{Frequency and Voltage Regulation}

Fig. 7 indicates that the primary deviation (without secondary control) after step changes in active power at $0.4 \mathrm{~s}$ and $0.7 \mathrm{~s}$ is $0.4 \mathrm{~Hz}$ (see middle figure). This deviation was effectively nullified to zero through secondary control (see lower figure). We can see that VSG-based secondary control gives superior results than conventional droop: both higher steady state performance and accurate nominal frequency tracking.

In Fig. 7(b), the primary control level responded to the sudden load changes with $0.7 \%$ voltage deviation (see middle figure). However, after activating secondary control, the response was significantly different: $0.02 \%$ transient overshoot which was regulated to zero within $20 \mathrm{~ms}$. This result was comparable for both droop and VSG-based controllers.

\section{Communication Link Latency}

The latencies in microgrid communication links are usually less than $100 \mathrm{~ms}$ [25]. In this study, the impact of communication delays of $100 \mathrm{~ms}$ to $150 \mathrm{~ms}$ were chosen. Fig. 9 shows that for communication delays up to $100 \mathrm{~ms}$ the proposed controller was able to maintain the same control performance of frequency and power sharing as with zero delay. The controller also maintained moderate levels of ripples for delays up to $120 \mathrm{~ms}$ (not shown to reduce figure clutter). However, as the delay was increased to $150 \mathrm{~ms}$ (Fig. 9), significantly higher active and reactive power ripples were recorded, while frequency performance was excellently sustained. Compared with results reported in [25], which showed accurate performance for communication latencies up to $1 \mathrm{~s}$, the control scheme in our study lost tracking accuracy and stability after $150 \mathrm{~ms}$ delay. Thus, the proposed method is robust to a limit of $150 \mathrm{~ms}$ latency.

\section{E. ROCOF Control}

Fig. 10 shows the ROCOF plots for secondary frequency regulation utilizing conventional droop and VSG inertia emulation. In particular, the transient performances are compared for power loop low-pass filter cut-off frequencies of $150 \mathrm{~Hz}$ and $1 \mathrm{kHz}$. As the filter frequencies increase, for the same step changes in load at $0.4 \mathrm{~s}$ and $0.7 \mathrm{~s}$, the magnitude of ROCOF also increases for both linear and MPC-based VSCs. What is more, for the applied load changes in this study, MPC is characterized by higher ROCOF than linear control: up to 2.3 times during steady state and up to 15 times more during transients. Specifically, during steady state, we observed 1.7 times more at $150 \mathrm{~Hz}$ and 2.3 times more at $1 \mathrm{kHz}$; and after step changes we observed 15 times more at $150 \mathrm{~Hz}$, and 10.5 times more at $1 \mathrm{kHz}$ - all for respective filter frequencies. 




(a) Frequency response of the secondary controller to step changes in active load. Top to bottom: active power sharing, primary frequency deviation, secondary frequency regulation.

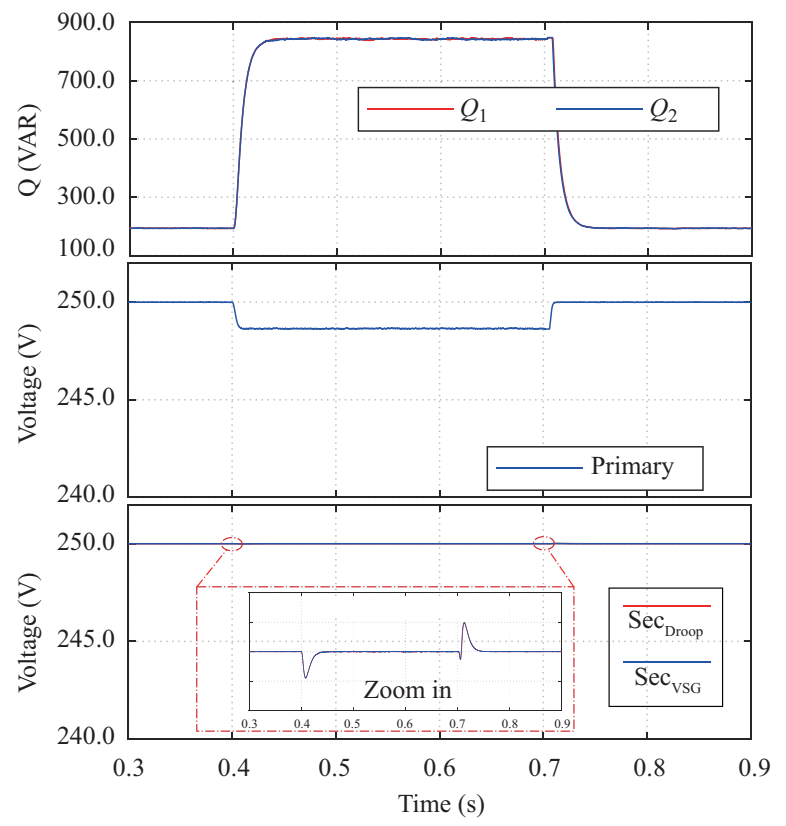

(b) Voltage response of the secondary controller to step changes in reactive load. Top to bottom: reactive power sharing, primary voltage deviation, secondary voltage regulation.

Fig. 7. Secondary frequency and voltage responses to load change -for equal values of inverter droop coefficients, inertia and line impedances. (a) Frequency response and (b) voltage response.

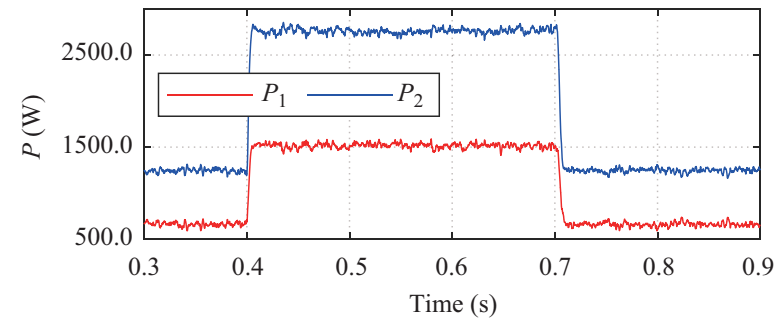

(a) Active power

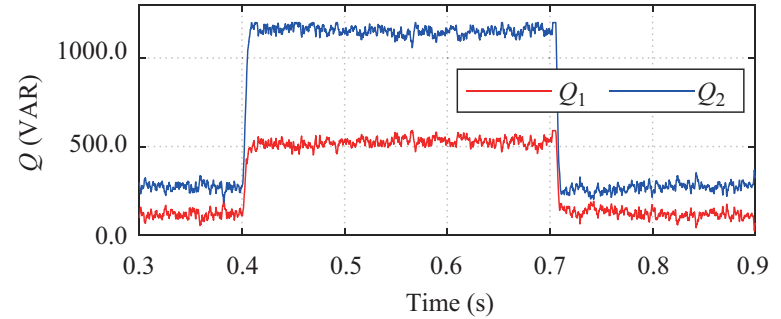

(b) Reactive power

Fig. 8. Power sharing performance with unequal values of inverter droop coefficients, inertia and line impedances. (a) Active power response and (b) reactive power response.

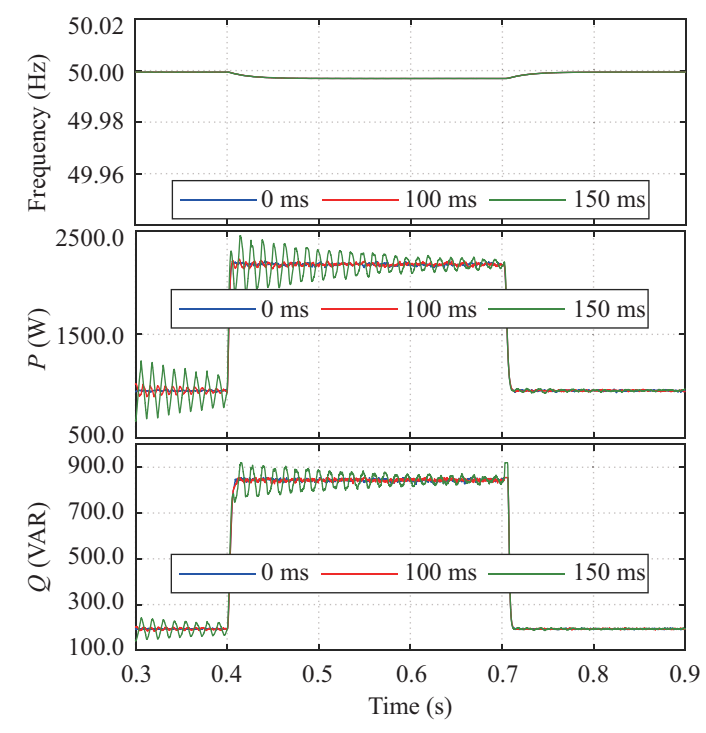

Fig. 9. Performance with communication delays. Top to bottom: frequency, active power and reactive power, respectively.
Nevertheless, despite the rising magnitude of ROCOF with increasing low-pass filter frequencies, the control scheme effectively reduced ROCOF - to zero during steady state and to about $2 \mathrm{~Hz} / \mathrm{s}$ during transients—-for all cases.

\section{CONCLUSION}

In this paper, the inertia emulation of VSG was complemented with the fast-dynamic properties of MPC in the distributed secondary regulation of frequency and voltage for an AC MG. This study shows that MPC has beneficial fast dynamic voltage response to sudden load changes. The proposed control scheme accurately regulates both the secondary frequency and voltage. In particular, although MPC improves the bandwidth for the regulation of voltage in a microgrid, high ROCOF values were recorded during sudden load-power changes. The ROCOF magnitude in the study also increased proportionally with both the magnitude of the disturbance and low-pass cut-off frequency in the power control loop. Therefore, the proposed control also reduces 


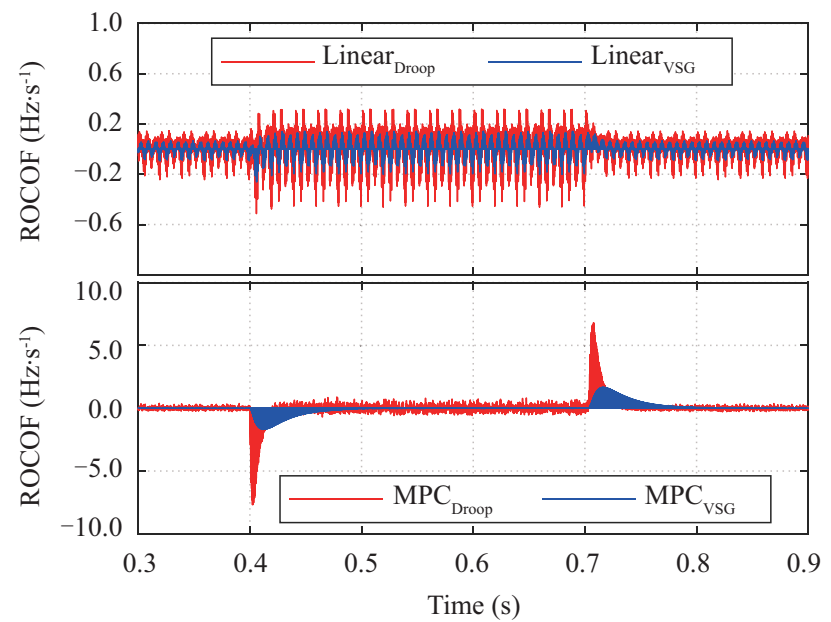

(a) $\omega_{\mathrm{f}}=150 \mathrm{~Hz}$

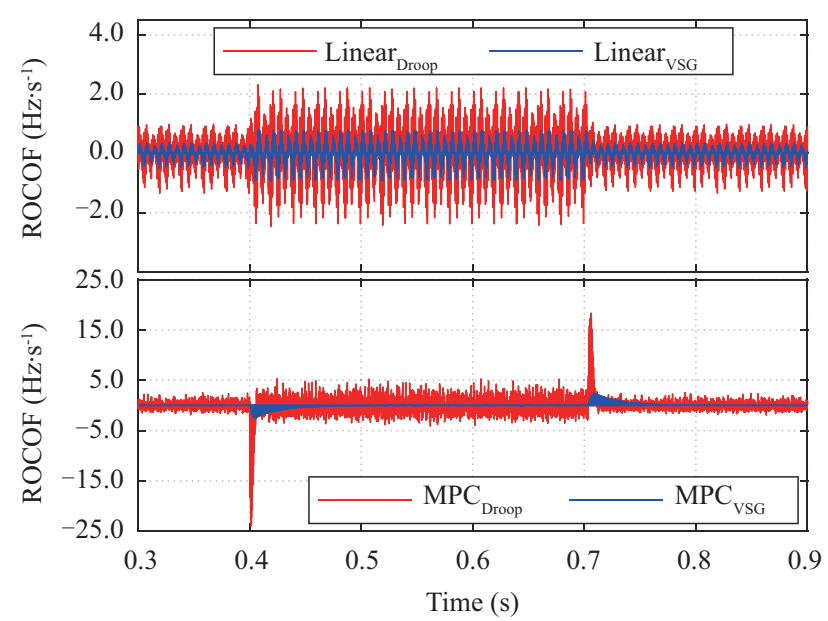

(b) $\omega_{\mathrm{f}}=1 \mathrm{kHz}$

Fig. 10. ROCOF plots for secondary regulation of frequency using Droop and VSG control. (a) ROCOF plot at $\omega_{\mathrm{f}}=150 \mathrm{~Hz}$ and (b) ROCOF plot at $\omega_{\mathrm{f}}=1 \mathrm{kHz}$.

the disturbance induced ROCOF to allowable bounds. Nevertheless, the accuracy of the proposed technique is limited to a communication latency that does not exceed $150 \mathrm{~ms}$.

\section{APPENDIX}

This section presents the small-signal analyses for inverter droop, VSG-emulation and the microgrid system. The method in [18] is extended to parallel-connected VSG-based inverters in a microgrid.

\section{A. Inverter Droop Small-Signal Analysis}

After a substitution of (6) and (7) into (4) and (5) respectively, and linearizing for small perturbations about the nominal values, we get the respective following expressions:

$$
\begin{aligned}
& \Delta \omega(s)=\frac{k_{p} \omega_{\mathrm{f}}}{s+\omega_{\mathrm{f}}} \Delta P(s), \\
& \Delta V(s)=\frac{k_{v} \omega_{\mathrm{f}}}{s+\omega_{\mathrm{f}}} \Delta Q(s) .
\end{aligned}
$$

These have the respective state-space forms

$$
\begin{array}{r}
\Delta \dot{\omega}=-\omega_{\mathrm{f}} \Delta \omega-k_{p} \omega_{\mathrm{f}} \Delta P, \\
\Delta \dot{V}=-\omega_{\mathrm{f}} \Delta V-k_{v} \omega_{\mathrm{f}} \Delta Q,
\end{array}
$$

where $\Delta x=$ small deviation of $x$ about the steady-state point, and $\dot{x}=$ time derivative of $x, \forall x \in\{\omega, V\}$. Given that

$$
\bar{V}=|V|(\cos \delta+j \sin \delta),
$$

in the $d q$ reference frame, the following apply, namely, $v_{d}=$ $|V| \cos \delta, v_{q}=|V| \sin \delta$, and

$$
\delta=\tan ^{-1}\left(\frac{v_{q}}{v_{d}}\right) .
$$

The linearized form of (27) is [18]:

$$
\Delta \delta=\frac{\partial \delta}{\partial v_{d}} \Delta v_{d}+\frac{\partial \delta}{\partial v_{q}} \Delta v_{q}
$$

The final state-space representations are expressed as:

$$
\begin{gathered}
\Delta \omega=s \Delta \delta(s)=m_{d} \Delta \dot{v}_{d}+m_{q} \Delta \dot{v}_{q}, \\
\Delta \dot{V}=n_{d} \Delta \dot{v}_{d}+n_{q} \Delta \dot{v}_{q},
\end{gathered}
$$

where $m_{d}=-v_{q} /\left(v_{d}^{2}+v_{q}^{2}\right), m_{q}=v_{d} /\left(v_{d}^{2}+v_{q}^{2}\right), n_{d}=v_{d} / \sqrt{v_{d}^{2}+v_{q}^{2}}$, and $n_{q}=v_{q} / \sqrt{v_{d}^{2}+v_{q}^{2}}$.

The voltage small-signal models are:

$\Delta \dot{v}_{d}=\frac{1}{n_{q} m_{d}-n_{d} m_{q}}\left(n_{q} \Delta \omega+\omega_{\mathrm{r}} n_{d} m_{q} \Delta v_{d}+\omega_{\mathrm{r}} n_{q} m_{q} \Delta v_{q}+k_{v} \omega_{\mathrm{r}} m_{q} \Delta Q\right)$,

$\Delta \dot{v}_{q}=-\frac{1}{n_{q} m_{d}-n_{d} m_{q}}\left(n_{d} \Delta \omega+\omega_{\mathrm{f}} n_{d} m_{q} \Delta v_{d}+\omega_{\mathrm{f}} n_{q} m_{d} \Delta v_{q}+k_{v} \omega_{\mathrm{f}} m_{d} \Delta Q\right)$.

\section{B. VSG-Emulation Small-Signal Analysis}

In order to achieve the VSG frequency-active power smallsignal relationship, a substitution of (3) into (2) gives

$$
k_{\mathrm{H}} \omega s=P_{n}-\tilde{P}-k_{d p}\left(\omega-\omega^{*}\right),
$$

where $k_{\mathrm{H}}=2 \mathrm{H}$, and $k_{d p}=k_{d}+k_{p}$. Accounting for the impact of the LPF on the active power,

$$
k_{\mathrm{H}} \omega s=P_{n}-\frac{\omega_{\mathrm{f}}}{s+\omega_{\mathrm{f}}} P-k_{d p}\left(\omega-\omega^{*}\right),
$$

which can be rewritten as 


$$
\left(\omega-\omega^{*}\right)=\frac{1}{k_{d p}}\left(P_{n}-\frac{\omega_{\mathrm{f}}}{s+\omega_{\mathrm{f}}} P-k_{\mathrm{H}} \omega s\right) .
$$

Linearizing (35),

$$
\Delta \omega=\frac{1}{k_{d p}}\left(-\frac{\omega_{\mathrm{f}}}{s+\omega_{\mathrm{f}}} \Delta P-k_{\mathrm{H}} \Delta \omega s\right) .
$$

Multiplying (36) by $k_{d p}\left(s+\omega_{\mathrm{f}}\right)$, we have

$$
\left(k_{d p}+k_{\mathrm{H}} \omega_{\mathrm{f}}\right) \Delta \omega s=-k_{d p} \omega_{\mathrm{f}} \Delta \omega-\omega_{\mathrm{f}} \Delta P .
$$

The second-order term in (37) is approximated to zero since the second-order coefficient $<<$ first-order coefficients. In statespace form, (37) becomes

$$
\Delta \dot{\omega}=-k_{d p} k_{\omega} \Delta \omega-k_{\omega} \Delta P,
$$

where $k_{\omega}=\omega_{\mathrm{f}} /\left(k_{d p}+k_{\mathrm{H}} \omega_{\mathrm{f}}\right)$. On the other hand, the VSG voltagedroop relationship is derived by first subsituting (7) into (5) as follows:

$$
\left(V-V^{*}\right)=-k_{v} \frac{\omega_{\mathrm{f}}}{s+\omega_{\mathrm{f}}}\left(\tilde{Q}-Q^{*}\right) .
$$

After linearization, the latter results in the following statespace form:

$$
\Delta \dot{V}=-\omega_{\mathrm{f}} \Delta V-k_{v} \omega_{\mathrm{f}} \Delta Q .
$$

A substitution of (29) into (38), gives

$$
\Delta \dot{\omega}=-k_{d p} k_{\omega}\left(m_{d} \Delta \dot{v}_{d}+m_{q} \Delta \dot{v}_{q}\right)-k_{\omega} \Delta P .
$$

Further substitutions in (41) for $\dot{v}_{d}$ and $\dot{v}_{q}$ from (31) and (32) respectively, and factorization give

$$
\Delta \dot{\omega}=-\frac{k_{d p} k_{\omega}}{n_{q} m_{d}-n_{d} m_{q}}\left[\left(m_{d} n_{q}-m_{q} n_{d}\right) \Delta \omega-k_{\omega} \Delta P\right] .
$$

(31), (32) and (42) can be combined into the following statespace model, viz.,

$$
\begin{aligned}
& {\left[\begin{array}{c}
\Delta \dot{\omega} \\
\Delta \dot{v}_{d} \\
\Delta \dot{v}_{q}
\end{array}\right]=\boldsymbol{E}\left[\begin{array}{l}
\Delta \omega \\
\Delta v_{d} \\
\Delta v_{q}
\end{array}\right]+\boldsymbol{F}\left[\begin{array}{c}
\Delta P \\
\Delta Q
\end{array}\right]} \\
& \text { where } \boldsymbol{E}=\left[\begin{array}{ccc}
-\frac{k_{d p} k_{\omega}}{n_{q} m_{d}-n_{d} m_{q}} & 0 & 0 \\
\frac{n_{q}}{n_{q} m_{d}-n_{d} m_{q}} & \frac{\omega_{\mathrm{f}} n_{d} m_{q}}{n_{q} m_{d}-n_{d} m_{q}} & \frac{\omega_{\mathrm{f}} n_{q} m_{q}}{n_{q} m_{d}-n_{d} m_{q}} \\
-\frac{n_{d}}{n_{q} m_{d}-n_{d} m_{q}} & -\frac{\omega_{\mathrm{f}} n_{d} m_{d}}{n_{q} m_{d}-n_{d} m_{q}} & -\frac{\omega_{\mathrm{f}} n_{q} m_{d}}{n_{q} m_{d}-n_{d} m_{q}}
\end{array}\right]
\end{aligned}
$$

[1] J. Fang, R. Zhang, H. Li, and Y. Tang, "Frequency derivative-based inertia enhancement by grid-connected power converters with a frequencylocked-loop," in IEEE Transactions on Smart Grid, vol. 10, no. 5, pp. 4918-4927, Sep. 2018.

[2] L. Miao, J. Wen, H. Xie, C. Yue, and W. Lee, "Coordinated control strategy of wind turbine generator and energy storage equipment for frequency support," in IEEE Transactions on Industry Applications, vol. 51, no. 4, pp. 2732-2742, Jul.-Aug. 2015.

[3] S. Yang, J. Fang, Y. Tang, H. Qiu, C. Dong, and P. Wang, "Synthetic inertia-based modular multilevel converter frequency control for improved microgrid frequency regulation," in Proceedings of 2018 IEEE Energy Conversion Congress and Exposition (ECCE), Portland, OR, 
2018, pp. 5177-5184.

[4] J. Fang, Y. Tang, H. Li, and X. Li, "A battery/ultra capacitor hybrid energy storage system for implementing the power management of virtual synchronous generators," in IEEE Transactions on Power Electronics, vol. 33, no. 4, pp. 2820-2824, Apr. 2017.

[5] J. Fang, H. Li, Y. Tang, and F. Blaabjerg, "Distributed power system virtual inertia implemented by grid-connected power converters," in IEEE Transactions on Power Electronics, vol. 33, no. 10, pp. 8488-8499, Oct. 2018.

[6] Y. Wang, M. Yu, and Y. Li, "Self-adaptive inertia control of DC microgrid based on fast predictive converter regulation," in IET Renewable Power Generation, vol. 11, no. 8, pp. 1295-1303, 2017.

[7] J. Jongudomkarn, J. Liu, and T. Ise, "Virtual synchronous generator control with reliable fault ride-through ability: A solution based on finiteset model predictive control," in IEEE Journal of Emerging and Selected Topics in Power Electronics, vol. 8, no. 4, pp. 3811-3824, Dec. 2020.

[8] O. Stanojev, U. Markovic, P. Aristidou, G. Hug, D. S. Callaway, and E. Vrettos, "MPC-based fast frequency control of voltage source converters in low-inertia power systems," in IEEE Transactions on Power Systems (Early Access), pp. 1-1, 03 Jun. 2020.

[9] U. Tamrakar, T. M. Hansen, R. Tonkoski, and D. A. Copp, "Mode predictive frequency control of low inertia microgrids," in Proceedings of 2019 IEEE 28th International Symposium on Industrial Electronics (ISIE), Vancouver, BC, Canada, 2019, pp. 2111-2116.

[10] K. T. Tan, X. Y. Peng, P. L. So, Y. C. Chu, and M. Z. Chen, "Centralized control for parallel operation of distributed generation inverters inmicrogrids," in IEEE Transactions on Smart Grid, vol. 3, no. 4, pp. 1977-1987, Dec. 2012.

[11] J. Liu, Y. Miura, and T. Ise, "Cost-function-based microgrid decentralized control of unbalance and harmonics for simultaneous bus voltage compensation and current sharing," in IEEE Transactions on Power Electronics, vol. 34, no. 8, pp. 7397-7410, Aug. 2019

[12] R. Heydari, Y. Khayat, M. Naderi, A. Anvari-Moghaddam, T. Dragicevic, and F. Blaabjerg, "A decentralized adaptive control method for frequency regulation and power sharing in autonomous microgrids," in Proceedings of 2019 IEEE 28th International Symposium on Industrial Electronics (ISIE), Vancouver, BC, Canada, 2019, pp. 2427-2432.

[13] A. Tavakoli, M. Negnevitsky, and K. M. Muttaqi, "A decentralized model predictive control for operation of multiple distributed generators in an islanded mode," in IEEE Transactions on Industry Applications,vol. 53, no. 2, pp. 1466-1475, Mar.-Apr. 2017.

[14] G. Lou, W. Gu, W. Sheng, X. Song, and F. Gao, "Distributed model predictive secondary voltage control of islanded microgrids with feedback linearization," in IEEE Access, vol. 6, pp. 50169-50178, Sep. 2018.

[15] K. Liu, T. Liu, Z. Tang, and D. J. Hill, "Distributed MPC-based frequency control in networked microgrids with voltage constraints," in IEEE Transactions on Smart Grid, vol. 10, no. 6, pp. 6343-6354, Nov. 2019.

[16] C. Ahumada, R. Cárdenas, D. Sáez, and J. M. Guerrero, "Secondary control strategies for frequency restoration in islanded microgrids with consideration of communication delays," in IEEE Transactions on Smart Grid, vol. 7, no. 3, pp. 1430-1441, May 2016.

[17] J. Liu, Y. Miura, and T. Ise, "Comparison of dynamic characteristics between virtual synchronous generator and droop control in inverter based distributed generators," in IEEE Transactions on Power Electronics, vol. 31, no. 5, pp. 3600-3611, May 2016.

[18] E. A. A. Coelho, P. C. Cortizo, and P. F. D. Garcia, "Small-signal stability for parallel-connected inverters in stand-alone AC supply systems," in IEEE Transactions on Industry Applications, vol. 38, no. 2, pp. 533-542, Mar-Apr. 2002

[19] J. Liu, Y. Miura, H. Bevrani, and T. Ise, "Enhanced virtual synchronous generator control for parallel inverters in microgrids," in IEEE Transactions on Smart Grid, vol. 8, no. 5, pp. 2268-2277, Sep. 2017.

[20] R. Heydari, T. Dragicevic, and F. Blaabjerg, "High-bandwidth secondary voltage and frequency control of VSC-based AC microgrid," in IEEE Transactions on Power Electronics, vol. 34, no. 11, pp. 11320-11331, Nov. 2019.

[21] C. Zheng, T. Dragicevic, and F. Blaabjerg, "Model predictive control based virtual inertia emulator for an islanded AC microgrid," in IEEE Transactions on Industrial Electronics(Early Access), pp. 1-1,10 Jul. 2020.
[22] J. C. Vasquez, J. M. Guerrero, M. Savaghebi, J. Eloy-Garcia, and R. Teodorescu, "Modeling, analysis, and design of stationary-referenceframe droop-controlled parallel three-phase voltage source inverters," in IEEE Transactions on Industrial Electronics, vol. 60, no. 4, pp. 12711280, Apr. 2013.

[23] O. Babayomi, Z. Zhang, and J. Kang, "High-bandwidth distributed virtual-inertia converters for AC microgrids," in Proceedings of 2020 IEEE PES/IAS Power Africa, Nairobi, Kenya, 2020, pp. 1-5.

[24] J. He, Y. W. Li, J. M. Guerrero, F. Blaabjerg, and J. C. Vasquez,“An islanding microgrid power sharing approach using enhanced virtual impedance control scheme," in IEEE Transactions on Power Electronics, vol. 28, no. 11, pp. 5272-5282, Nov. 2013.

[25] J. S. Gómez, D. Sáez, J. W. Simpson-Porco, and R. Cárdenas, "Distributed predictive control for frequency and voltage regulation in microgrids," in IEEE Transactions on Smart Grid, vol. 11, no. 2, pp. 1319-1329, Mar. 2019.

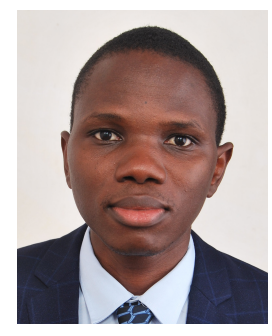

Oluleke Babayomi received the B.S. and M.S. degrees in electrical and electronics engineering from the University of Lagos, Nigeria in 2006 and 2016 respectively. Until 2019, he was a Principal Engineer, Senior Technical Assistant to the CEO of the Centre for Space Transport and Propulsion (CSTP), and software development Team Leader at the National Space Research and Development Agency, Nigeria (NASRDA). Since 2019, he has been a doctoral candidate of electrical engineering at Shandong University, China, sponsored by the China Scholarship Council Award.

Oluleke won the Best Technical Advance Award of the 2020 IEEE Smart Village -Meet the Entrepreneurs Competition, and was a recipient of the 2018 NASRDA-CSTP CEO's Commendation. His current research interests include model predictive control, power electronics for microgrids, sustainable energy systems and off-grid electrification.

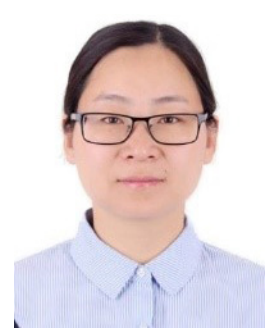

Zhen Li was born in Shandong, China, in 1983. She received the M.S. degree in material science from the State Key Laboratory of Crystal Materials, Shandong University, Jinan, China, in 2004, and the $\mathrm{Ph} . \mathrm{D}$. degree in semiconductor materials from the Institute of Crystallography and Structural Physics, Friedrich-Alexander University Erlangen-Nürnberg (FAU), Erlangen, Germany, in 2017. From 2014 to 2015, she was a Teaching and Research Assistant at FAU. Since 2018, she has been an Assistant Professor with Shandong Normal University, Jinan. From 2019 she has been an Associate Professor with Shandong University, Jinan. Her research interests include wideband-gap devices and power electronic converter reliability.

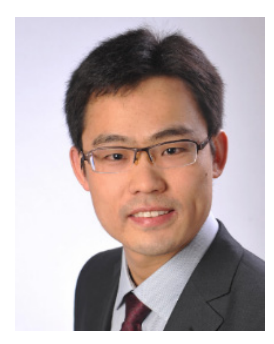

Zhenbin Zhang was born in Shandong, China, in 1984. He received the B.S. degree in Electrical Engineering and Automation at Harbin Engineering University, Harbin, China, in 2008. From 2008 to 2011, he studied control theory and engineering at Shandong University, Jinan, China. In 2016, he received the Ph.D. degree at the Institute for Electrical Drive Systems and Power Electronics (EAL), Technical University of Munich (TUM), Germany, "Summa cum laude". From 2016 to 2017, he worked as a Research Fellow and the Group Leader of "Modern control strategies for electrical drives" at EAL, TUM. Since 2017, he has been a full professor and director of the Lab for More Power Electronics and Energy Systems (MPEES) at Shandong University, Jinan, China.

Dr. Zhang received the VDE-Award, Suedbayern, Germany, in 2017. His research interests include power electronics and electrical drives, sustainable energy systems, smart and microgrids. 